\title{
Evidence for a Clathrin-independent mode of endocytosis at a continuously active sensory synapse
}

\author{
Michaela Fuchs, Johann Helmut Brandstätter and Hanna Regus-Leidig*
}

Animal Physiology, Department of Biology, Friedrich-Alexander-University Erlangen-Nuremberg, Erlangen, Germany

\section{Edited by:}

Dieter Wicher, Max Planck Institute for Chemical Ecology, Germany

\section{Reviewed by:}

Martin Heine, Leibniz Institute for Neurobiology, Germany

Wallace B. Thoreson, University of

Nebraska Medical Center, USA

\section{*Correspondence:}

Hanna Regus-Leidig, Animal

Physiology, Department of Biology, Friedrich-Alexander-University

Erlangen-Nuremberg, Staudtstraße 5, 91058 Erlangen, Germany

e-mail: hanna.regus-leidig@fau.de
Synaptic vesicle exocytosis at chemical synapses is followed by compensatory endocytosis. Multiple pathways including Clathrin-mediated retrieval of single vesicles, bulk retrieval of large cisternae, and kiss-and-run retrieval have been reported to contribute to vesicle recycling. Particularly at the continuously active ribbon synapses of retinal photoreceptor and bipolar cells, compensatory endocytosis plays an essential role to provide ongoing vesicle supply. Yet, little is known about the mechanisms that contribute to endocytosis at these highly complex synapses. To identify possible specializations in ribbon synaptic endocytosis during different states of activity, we exposed mice to controlled lighting conditions and compared the distribution of endocytotic proteins at rod and cone photoreceptor, and ON bipolar cell ribbon synapses with light and electron microscopy. In mouse ON bipolar cell terminals, Clathrin-mediated endocytosis seemed to be the dominant mode of endocytosis at all adaptation states analyzed. In contrast, in mouse photoreceptor terminals in addition to Clathrin-coated pits, clusters of membranously connected electron-dense vesicles appeared during prolonged darkness. These clusters labeled for Dynamin3, Endophilin1, and Synaptojanin1, but not for AP180, Clathrin LC, and hsc70. We hypothesize that rod and cone photoreceptors possess an additional Clathrinindependent mode of vesicle retrieval supporting the continuous synaptic vesicle supply during prolonged high activity.

\section{Keywords: ribbon synapse, endocytosis, active zone, dynamin, endophilin, synaptojanin, piccolino, bassoon}

\section{INTRODUCTION}

At chemical synapses, exocytosis of transmitter-filled synaptic vesicles is followed by compensatory endocytosis to retrieve synaptic vesicle components from the plasma membrane and to restore the cell surface area. Multiple pathways contribute to compensatory endocytosis at chemical synapses, including Clathrin-mediated retrieval of single vesicles, bulk retrieval of large cisternae, and kissand-run retrieval (reviewed in Dittman and Ryan, 2009; Royle and Lagnado, 2010; Haucke et al., 2011; Saheki and de Camilli, 2012). In contrast to conventional chemical synapses, tonically active ribbon synapses support high and sustained rates of exocytosis (reviewed in Matthews and Fuchs, 2010; Mercer and Thoreson, 2011; Regus-Leidig and Brandstätter, 2012). Thus, compensatory endocytosis has to play an important role for vesicle replenishment at these synapses.

Among the different types of sensory neurons with ribbon synapses, photoreceptors display extraordinary high rates of sustained glutamate release, with highest release rates in the dark (Heidelberger et al., 2005). The ultrastructural hallmark of photoreceptor synapses, the presynaptic ribbon, is an electron-dense plate-like structure which extends several hundred $\mathrm{nm}$ into the cytoplasm and tethers a large number of transmitter vesicles via fine filaments (Rao-Mirotznik et al., 1995; Sterling and Matthews, 2005; tom Dieck and Brandstätter, 2006). The exact function of the synaptic ribbon in photoreceptor neurotransmission is not yet fully understood, but it is widely accepted that the synaptic ribbon ensures the precise spatial and temporal control of vesicle fusion, and accommodates the high rates of synaptic transmission (Schmitz, 2009; Regus-Leidig and Brandstätter, 2012).

Studies on the mechanisms of endocytosis at photoreceptor ribbon synapses are rare, but there is growing evidence for Clathrin-mediated endocytosis (CME) as a predominant mechanism. Early tracer uptake studies on cone photoreceptors from different species showed labeling of single vesicles, some of which were coated (reviewed in LoGiudice and Matthews, 2007), and a few electron microscopical studies on rat, guinea pig, and mouse photoreceptor terminals showed Clathrin-coated pits (CCP) or Clathrin-coated vesicles (CCV; Gray and Pease, 1971; Spiwoks-Becker et al., 2001; Bai et al., 2006; Zampighi et al., 2011). In addition, recent immunocytochemical studies on various species report the presence of Clathrin, Dynamin, Amphiphysin, Endophilin, and Synaptojanin in photoreceptor terminals (Sherry and Heidelberger, 2005; Xi et al., 2007; Holzhausen et al., 2009; Grossman et al., 2013; Wahl et al., 2013). All these studies were performed on light adapted retinae, when photoreceptors are minimally active. Since photoreceptors release the highest amounts of glutamate in the dark, it is conceivable that they developed specialized adaptations to increased vesicle turnover rates during darkness.

In this study, we exposed mice to a controlled light regime with light adaptation followed by different durations of dark exposure and compared the distribution of endocytotic proteins at rod photoreceptor, cone photoreceptor, and bipolar cell ribbon synapses in the differently adapted retinae with light and electron 
microscopy. Based on our findings, we propose that in addition to CME, photoreceptors possess a Clathrin-independent mode of compensatory vesicle retrieval during prolonged, maximal activity.

\section{MATERIALS AND METHODS ETHICS STATEMENT}

All animal experiments were approved and registered by the county's animal welfare authorities (permit number: 54-2531.3115/06; Regierung von Mittelfranken, Ansbach, Germany) and performed in compliance with the guidelines for the welfare of experimental animals issued by the Federal Government of Germany and the FAU Erlangen-Nuremberg. Mouse breeding was performed in the animal facilities of the FAU Erlangen-Nuremberg according to European and German (Tierschutzgesetz) guidelines for the welfare of experimental animals (AZ 820-8791. 2.63).

\section{ANIMALS AND LIGHT REGIME}

Adult (age 4 months) male Dark Agouti rats (kindly provided by the Franz-Penzoldt-Zentrum, Erlangen), adult (2-3 months) male and female C57BL/6JRj (BL/6) mice, Bsn $\Delta \mathrm{Ex} 4 / 5$ mice (Altrock etal., 2003; kindly provided by E. D. Gundelfinger, Leibniz Institute for Neurobiology, Magdeburg, Germany), Tg(Rac3-EGFP)JZ58Gsat/Mmcd (Rac3-EGFP) mice, and Tg(Lrrc55-EGFP)KS290Gsat/Mmcd (Lrrc55-EGFP) mice were used in this study. The latter two strains were obtained from the Mutant Mouse Regional Resource Center, a NCRR-NIH funded strain repository, and were donated to the MMRRC by the NINDS funded GENSAT BAC transgenic project. Both strains were back-crossed to a BL/6 background.

All animals were kept in a 12/12 h light/dark cycle with light on at 6:00 a.m. and an average illumination of 200 lux (white light; TLD 58W/25 tubes, Philips, Hamburg, Germany). Light and dark adaptation was performed as follows: mice were light adapted for $3 \mathrm{~h}$ or light adapted for $3 \mathrm{~h}$ and subsequently exposed to different periods of darkness ( $1 \mathrm{~min}, 15 \mathrm{~min}, 3 \mathrm{~h}, 15 \mathrm{~h}$ ).

\section{RETINA PREPARATION FOR LIGHT MICROSCOPY AND LIGHT MICROSCOPIC ANALYSIS}

Preparation of retinal tissue and antibody incubation for light microscopic immunocytochemistry (ICC) was performed as described previously (tom Dieck et al., 2005; Regus-Leidig et al., 2009). Dark adapted retinae were prepared and fixed under dim red light. Briefly, the eyes were opened and retinae were immersion fixed in the eyecup for 15 or $30 \mathrm{~min}$ in $4 \%$ paraformaldehyde (PFA) in phosphate buffer ( $\mathrm{PB} ; 0.1 \mathrm{M}, \mathrm{pH}$ 7.4). Retinae were mounted in freezing medium (Reichert-Jung, Bensheim, Germany), and $12 \mu \mathrm{m}$ thick vertical sections were cut with a cryostat (Leica CM3050 S, Leica, Wetzlar, Germany). Primary antibody incubation was carried out overnight at room temperature, secondary antibody incubation for $1 \mathrm{~h}$. For analysis, labeled sections were examined with a Zeiss Axio Imager Z2 equipped with an ApoTome (Zeiss, Oberkochen, Germany). Images were taken with a $20 \times(0.8$, Apochromat $)$ or a $100 \times$ (1.3 oil, Plan-Neofluar) objective as stacks of multiple optical sections, and projections were calculated with the ZEN blue
2012 software (Zeiss). Images were adjusted for contrast and brightness using Adobe Photoshop CS (Adobe, San Jose, CA, USA). 3D reconstructions from $\mathrm{Z}$-stacks were generated with Imaris software (Bitplane, Zurich, Switzerland). Colocalization of immunofluorescent puncta was determined by using the custommade analyzing software "OpenView" on projections of z-section stacks. Rectangular regions $(4 \times 4$ pixel $)$ centered on individual Dynamin-puncta were automatically drawn and compared with the fluorescent staining of the respective second channel (i.e., staining for Endophilin or Synaptojanin1; software written by N. E. Ziv, The Rappaport Family Institute for Research in Medical Sciences, Department of Physiology and Biophysics, and The Lorry Lokey Interdisciplinary Center for Life Sciences and Engineering, Technion-Israel Institute of Technology, Haifa, Israel; Regus-Leidig et al., 2010b). For each adaptation state and antibody combination, 3 images from 3 retinal sections per mouse $(n=3)$ were analyzed.

\section{RETINA PREPARATION FOR ELECTRON MICROSCOPY}

For conventional electron microscopy, retinae were fixed in $4 \%$ PFA and $2.5 \%$ glutaraldehyde for $2 \mathrm{~h}$ at room temperature. Tissue contrasting was carried out by incubation in $1.5 \%$ potassium ferrocyanide and $2 \%$ osmium tetroxide in $0.1 \mathrm{M}$ cacodylate buffer ( $\mathrm{pH} 7.4$ ) for $1.5 \mathrm{~h}$. Retinae were dehydrated using an ethanol series and propylene oxide with $0.5 \%$ uranyl acetate added at the $70 \%$ ethanol step. The tissue was embedded in Renlam resin (Serva, Heidelberg, Germany).

For pre-embedding immunoelectron microscopy, retinae were prefixed in $4 \%$ PFA in Soerensen buffer $\left(0.1 \mathrm{M} \mathrm{Na}_{2} \mathrm{HPO}_{4} \cdot 2 \mathrm{H}_{2} \mathrm{O}\right.$, $0.1 \mathrm{M} \mathrm{KH}_{2} \mathrm{PO}_{4}, \mathrm{pH} 7.4$ ) for $50 \mathrm{~min}$ at room temperature and further processed as described previously (Brandstätter et al., 1996). Briefly, after four cycles of freezing in liquid nitrogen and thawing at $37^{\circ} \mathrm{C}$, retinae were PBS washed and embedded in buffered 2\% Agar. Agar blocks were sectioned in $100 \mu \mathrm{m}$ sections with a vibratome (Leica VT 1000 S, Leica, Wetzlar, Germany). The sections were incubated in $10 \%$ normal goat serum, $1 \%$ bovine serum albumin in PBS for $2 \mathrm{~h}$, followed by incubation with primary antibodies for 4 days at $4^{\circ} \mathrm{C}$. PBS washed sections were incubated with biotinylated secondary antibodies, and visualized by Vectastain ABC-Kit (both from Vector Laboratories, Burlingame, CA, USA). The sections were fixed in 2.5\% glutaraldehyde in cacodylate buffer. Diaminobenzidine precipitates were silver enhanced and postfixed in $(1.5 \%$ potassium ferrocyanide and) $0.5 \% \mathrm{OsO}_{4}$ in cacodylate buffer for $30 \mathrm{~min}$ at $4^{\circ} \mathrm{C}$. Dehydrated specimens were flat-mounted in Epon resin (Fluka, Buchs, Switzerland).

Ultrathin sections $(58 \mathrm{~nm})$ were stained with uranyl acetate and lead citrate. The sections were examined and photographed using a Zeiss EM10 electron microscope (Zeiss, Oberkochen, Germany) and a Gatan SC1000 Orius TM CCD camera (GATAN, Munich, Germany) in combination with the Digital Micrograph 3.1 software (GATAN, Pleasanton, CA, USA). Images were adjusted for contrast and brightness using Adobe Photoshop CS5 (Adobe, San Jose, CA, USA). 3D reconstructions from serial sections were created with Reconstruct v1.1.0.0 (J. Fiala, Medical College of Georgia). Vesicle diameters were measured using ImageJ (Schneider et al., 2012). 


\section{ANTIBODIES}

The following antibodies were used for ICC and pre-embedding immunoelectron microscopy (pre-EM): monoclonal mouse antiCalbindin (ICC 1:2,000; \#214011 Synaptic Systems, Göttingen, Germany), mouse anti-Clathrin light chain (Clathrin LC; ICC 1:1,000; \#113011 Synaptic Systems), mouse anti-Dynamin (ICC 1:10,000; \#ADI-VAM-SV041-E Enzo Life Sciences, Farmingdale, NY, USA), mouse anti-uncoating ATPase (hsc70; ICC 1:100; \#149011 Synaptic Systems), polyclonal rabbit anti-Amphiphysin (ICC/pre-EM 1:10,000; \#120002 Synaptic Systems), rabbit antiAP180 (ICC 1:10,000; \#155003 Synaptic Systems), rabbit antiCaveolin1 (ICC 1:1000; \#161003 Synaptic Systems), rabbit anti-Dynamin3 (ICC/pre-EM 1:10,000; \#115302 Synaptic Systems), rabbit anti-Endophilin1 (ICC/pre-EM 1:10,000; \#159002 Synaptic Systems), rabbit anti-Synaptojanin1 (ICC 1:5,000; preEM 1:10,000; \#145003 Synaptic Systems), rabbit anti-RIBEYE A-domain (ICC 1:50,000; \#192103 Synaptic Systems; RegusLeidig etal., 2013), rabbit anti-Velis-3 (ICC 1:5,000, \#515600 Invitrogen/Life technologies, Carlsbad, CA, USA; RegusLeidig etal., 2010a), guinea pig anti-GFP (ICC 1:400; this antibody was generated and affinity-purified as described in Mühlhans etal., 2011. Peptide immunization of guinea pigs was performed by Pineda Antikörper-Service, Berlin, Germany), guinea pig anti-Piccolo 44a (ICC 1:4,000; Dick et al., 2001), guinea pig anti-VGluT1 (ICC 1:50,000, \#AB5905 Millipore; Regus-Leidig etal., 2009). All Synaptic Systems antibodies were tested for specificity by the manufacturer, and western blot images and immunocytochemical stainings showing the specificity are available on the Synaptic Systems website.

The following secondary antibodies were used: Alexa ${ }^{T M} 488$ goat anti-guinea pig, goat anti-mouse and goat anti-rabbit IgG conjugates (1:500; Molecular Probes, Eugene, OR, USA), Cy3 goat anti-mouse and goat anti-rabbit IgG conjugates (1:200; Dianova, Hamburg, Germany), Cy5 goat anti-guinea pig, goat anti-mouse and goat anti-rabbit IgG conjugates (1:100; Dianova).

\section{RESULTS \\ DISTRIBUTION OF ENDOCYTOTIC PROTEINS IN THE MOUSE RETINA}

First, we stained vertical cryostat sections of light adapted C57BL/6JRj (BL/6) retinae for proteins involved in various modes of endocytosis, but particularly for proteins described in the different steps of CME (for a detailed review, see e.g., Saheki and de Camilli, 2012), i.e., nucleation (AP180, Clathrin LC; Figures 1A,D), budding and scission (Endophilin, Amphiphysin, Synaptojanin, Dynamin; Figures 1B,E), and uncoating (hsc70; Figures 1C,F). For all examined endocytotic proteins, we observed staining in both synaptic layers of the retina, the outer (OPL) and the inner (IPL) plexiform layer. Strong staining was detected for Clathrin LC, Endophilin1, Amphiphysin1, and Dynamin3 (Figures 1D,E), weaker staining for AP180 (Figure 1D), Synaptojanin 1 (Figure 1E), and hsc70 (Figure 1F). Other modes than CME that are discussed for photoreceptor ribbon synapses include endocytosis via caveolae (Kachi et al., 2001; Kim et al., 2006). However, staining of retina sections with an antibody against Caveolin 1 did not produce any specific staining in the two synaptic layers (data not shown).
For the detailed analysis of the presence and possible differences in the expression of CME components at different types of retinal ribbon synapses - rod photoreceptor, cone photoreceptor, and ON bipolar cell synapses - we double labeled vertical cryostat sections of light adapted retinae for the endocytotic proteins and respective cell markers. Rod photoreceptor terminals of BL/6 mouse retina were stained with antibodies against Velis-3 or VGluT1 (Figure 2A), cone photoreceptors were visualized using retinae of Rac3-EGFP mice, which express eGFP in their cone photoreceptors (Figure 2B), and ON bipolar cells were detected using retinae of Lrrc55-EGFP mice, which express eGFP in their ON bipolar cells (Figure 2C; Regus-Leidig et al., 2013). EGFP of transgenic retinae was intensified with an antibody against GFP.

The majority of analyzed endocytotic components was present and diffusely distributed in all three types of ribbon synapses, i.e., in terminals of rod (Figure 2D) and cone (Figure 2E) photoreceptors, and in ON bipolar cell terminals (Figure 2F). Synaptojanin1 was the only protein which was almost undetectable in light adapted rod and cone photoreceptor terminals (Figures 2D,E); in ON bipolar cell terminals it was diffusely distributed (Figure 2F). Although some of these proteins might also be involved in other modes of endocytosis (Nichols and Lippincott-Schwartz, 2001; Jockusch etal., 2005; Llobet et al., 2011), from our findings we conclude that CME is an important contributor to endocytosis at retinal ribbon synapses with only little variation between photoreceptors and ON bipolar cells regarding the analyzed protein variants. However, immunolabeling for AP180 and Clathrin LC appeared weaker in cone terminals than in rod terminals (Figures 2D,E). This hints at possible rod-cone differences in the expression of these proteins and thus may distinguish the two photoreceptor types' dependence on CME for synaptic vesicle retrieval. Evidence that endocytosis in salamander rods and cones involves different mechanisms has recently been provided by Van Hook and Thoreson (2012).

\section{ULTRASTRUCTURAL ANALYSIS OF CME IN PHOTORECEPTOR RIBBON SYNAPSES}

In contrast to the diffuse distribution of endocytotic proteins in our light microscopical analysis (Figure 2), a ring-shaped concentration of Dynamin around the rod photoreceptor synaptic ribbon corresponding to a circular hotspot of endocytosis has been reported (Wahl et al., 2013). To analyze the distribution of CCP in relation to photoreceptor synaptic ribbons in light adapted retinae, we performed an ultrastructural analysis of rod and cone photoreceptor ribbon synapses in the BL/6 retina (Figure 3). CCP (Figures 3A,B; dashed circles and high power view insets) were found in the periactive zones at various distances from the synaptic ribbons (arrowhead) in both rod and cones. To better judge the distribution of CCP in the periactive zone, we 3Dreconstructed rod photoreceptor synaptic terminals $(n=3)$ from serial ultrathin sections. For reconstruction we did not differentiate between the different stages of CCP, and reconstructed all Clathrin-coated structures from the nucleation stage to the scission stage (Figure 3C). Figure 3D shows a representative example of a light adapted rod photoreceptor ribbon synaptic complex viewed from three different angles rotated by $90^{\circ}$ : 


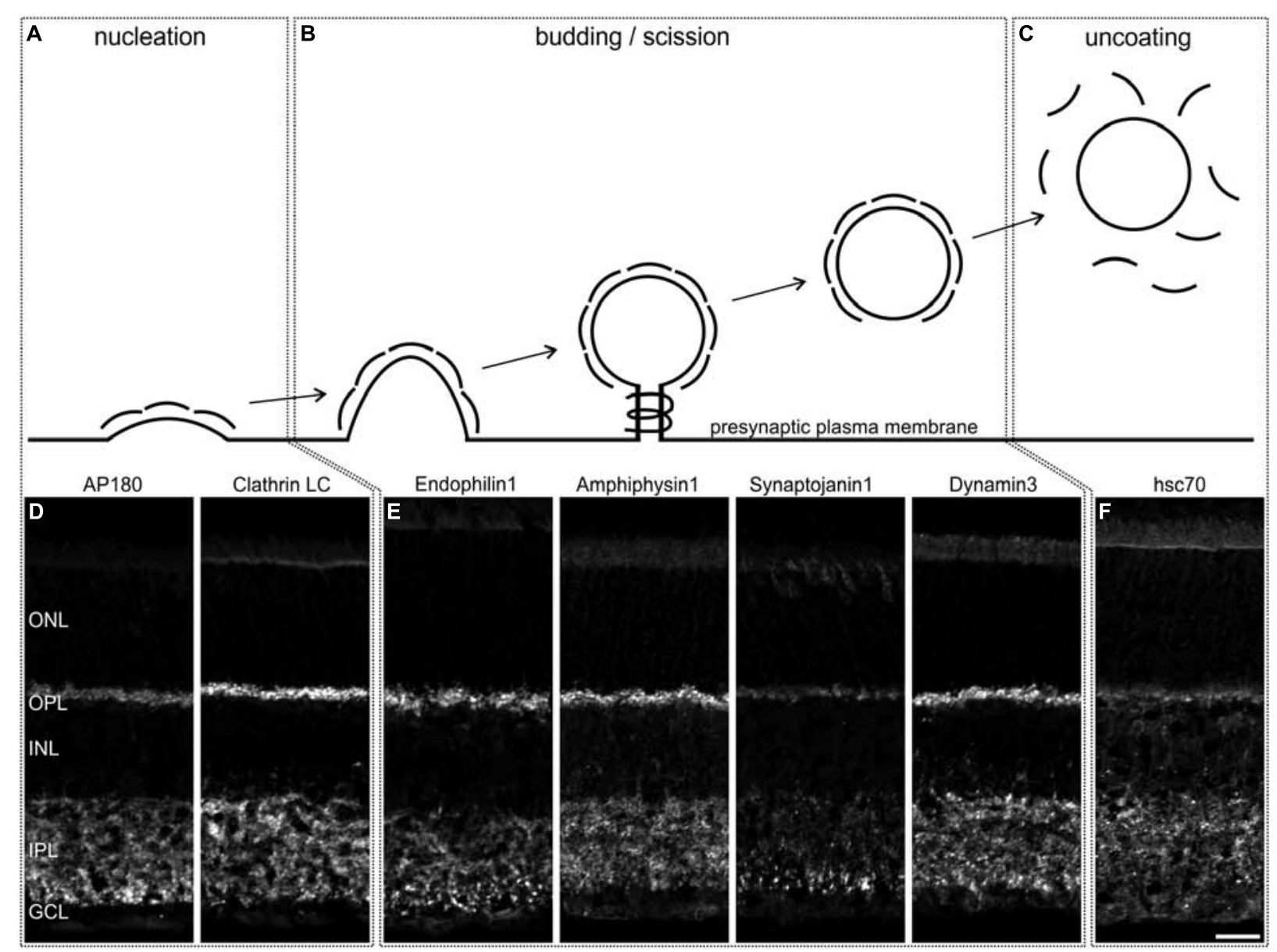

FIGURE 1 | Distribution of endocytotic proteins in a $3 \mathrm{~h}$ light adapted mouse retina. (A-C) Schematic representation of the steps of Clathrin-mediated endocytosis. (D-F) Images of vertical cryostat sections through light adapted $\mathrm{C} 57 \mathrm{BL} / 6 \mathrm{JRj}$ retinae stained with antibodies against endocytotic proteins involved in these steps. ONL, outer nuclear layer; $\mathrm{OPL}$, outer plexiform layer; $\mathbf{I N L}$, inner nuclear layer; IPL, inner plexiform layer; $\mathrm{GCL}$, ganglion cell layer. Scale bar: $20 \mu \mathrm{m}$. synaptic ribbon (black), tethered synaptic vesicles (blue spheres), invaginating postsynaptic elements (Boissonnat surfaces), and CCP (red spheres). CCP were frequently observed in the periactive zone, but never directly at the base of the synaptic ribbon. They appeared not to be enriched in a ring-shaped distribution around the synaptic ribbon, but were distributed all-over the plasma membrane of the invaginating postsynaptic elements (Figure 3D). Free CCV could rarely be seen in the cytoplasm of rod photoreceptor terminals (Figure 3C), most likely indicating rapid uncoating after scission (Brodin et al., 2000).

\section{ENDOCYTOTIC PROTEINS REDISTRIBUTE IN PHOTORECEPTOR TERMINALS FOLLOWING DARK EXPOSURE}

The reported enrichment of Dynamin in the periactive zone surrounding the photoreceptor synaptic ribbon complex (Wahl et al., 2013) is at odds both with our light microscopical finding of a diffuse distribution of endocytotic proteins including Dynamin3 (Figures 2D,E), and with the ultrastructural distribution of CCP in our 3D-reconstructed rod photoreceptor ribbon synaptic complexes (Figure 3D). The different anti-Dynamin antibodies used in the study by Wahl et al. (2013) and our study are not the reason for the differing results. Wahl et al. (2013) used a monoclonal mouse anti-Dynamin 1 antibody generated against a peptide stretch in the C-terminal region of Dynamin1 that is also conserved in Dynamin2 and Dynamin3, thus most likely not distinguishing between the three Dynamin forms. In our study, we used two anti-Dynamin antibodies recognizing all three Dynamin forms and an anti-Dynamin3 specific antibody (Section Materials and Methods). The pan Dynamin antibodies and the anti-Dynamin3 antibody produced the same staining pattern in the immunocytochemical experiments, suggesting that Dynamin3 is the major Dynamin form at photoreceptor synaptic terminals.

To figure out what might be the reason for the discrepancy between our study and the study by Wahl et al. (2013), and since all our experiments so far had been performed on light adapted retinae, we examined the localization of endocytotic proteins following increasing periods of dark exposure, i.e., increasing synaptic activity. Mice were light adapted for $3 \mathrm{~h}$ and then dark adapted for $1 \mathrm{~min}, 15 \mathrm{~min}, 3 \mathrm{~h}$, and $15 \mathrm{~h}$. As shown in 

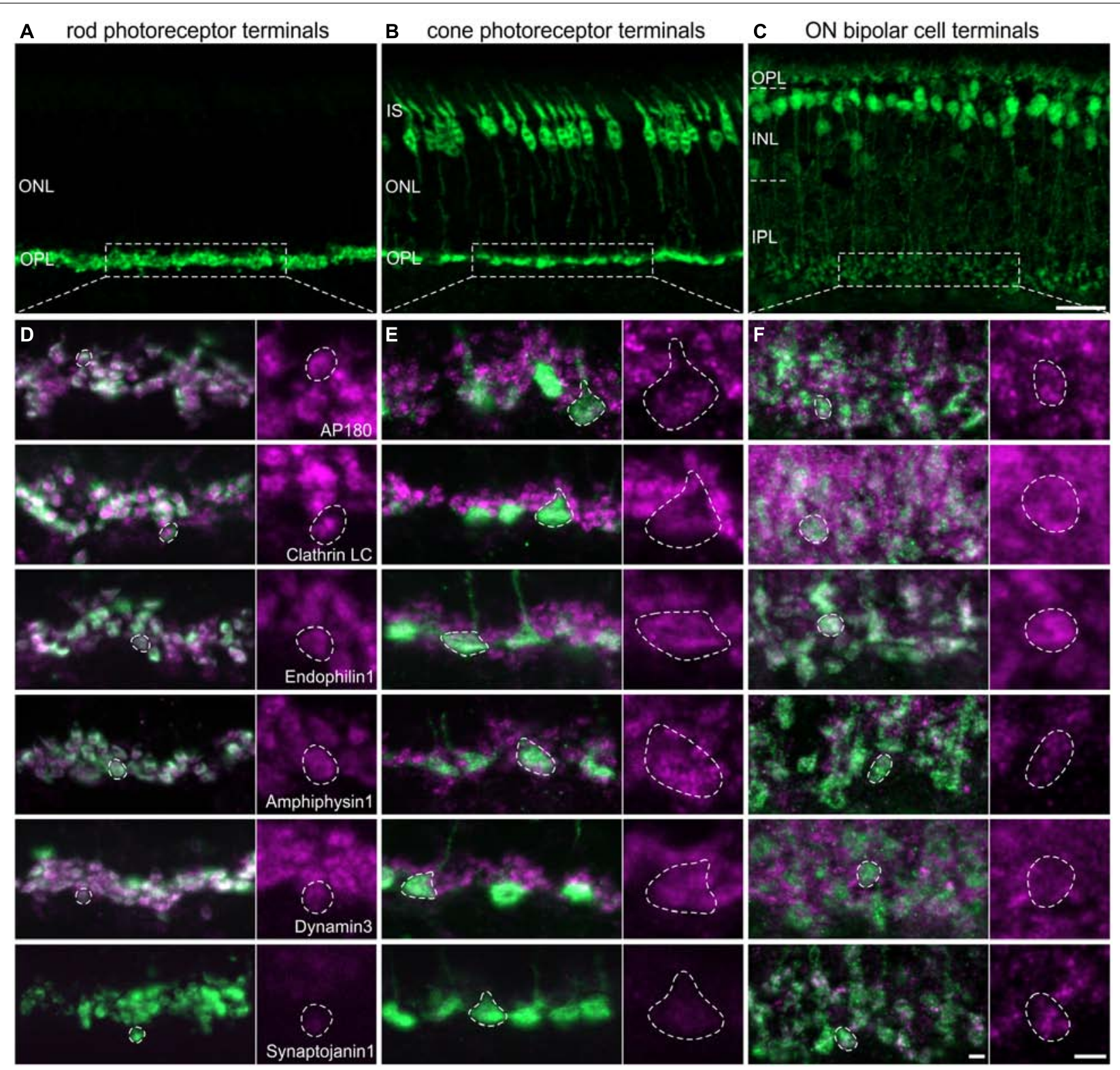

FIGURE 2 | Endocytotic proteins in terminals of $3 \mathrm{~h}$ light adapted mouse rod photoreceptors, cone photoreceptors, and ON bipolar cells. (A-C) Images of vertical cryostat sections through C57BL/6JRj (A), Rac3-EGFP (B), and Lrrc55-EGFP $(\mathbf{C})$ retinae stained with antibodies against VGluT1 $(\mathbf{A})$ and GFP (B,C). (D) Images of rod photoreceptor terminals of C57BL/6JRj retinae stained with antibodies against Velis-3 or VGluT1 (green) and endocytotic proteins (magenta). (E) Images of cone photoreceptor terminals of
Rac3-EGFP retina stained with antibodies against GFP (green) and endocytotic proteins (magenta). (F) Images of ON bipolar cell terminals of Lrrc55-EGFP retina stained with antibodies against GFP (green) and endocytotic proteins (magenta). Single synaptic terminals are delineated with dashed lines. ONL, outer nuclear layer; OPL, outer plexiform layer; IS, inner segments; INL, inner nuclear layer; IPL, inner plexiform layer. Scale bar in C (for $\mathbf{A}-\mathbf{C}$ ): $20 \mu \mathrm{m}$, in $\mathbf{F}$ (for $\mathbf{D}-\mathbf{F}$ ): $2 \mu \mathrm{m}$.
Figure 4A, Dynamin3 underwent a remarkable redistribution in the OPL in the dark adapted retinae; note that no obvious adaptation-dependent differences in Dynamin3 distribution were detected in the terminals of ON bipolar cells at any time point (data not shown). Already after 1 to $15 \mathrm{~min}$ of increased activity, Dynamin3 labeling changed from a diffuse to a circular appearance (Figure 4A), which is in line with the Dynamin distribution reported by Wahl et al. (2013). The same staining patterns after 1 and 15 min of darkness were observed for Endophilin1,
Amphiphysin1, Clathrin LC, and AP180 (data not shown), reflecting a clustering of endocytotic proteins in the periactive zone during periods of increased exocytosis.

The most striking redistribution of Dynamin3 was seen after prolonged periods of darkness ( 3 to $15 \mathrm{~h}$ ), when distinct Dynamin3 hotspots appeared throughout the entire OPL in the rod and cone photoreceptor synaptic terminals (Figures 4A,C,D). An antibody against VGluT1 was used to label both rod and cone photoreceptor terminals (Figure 4C). To be able 


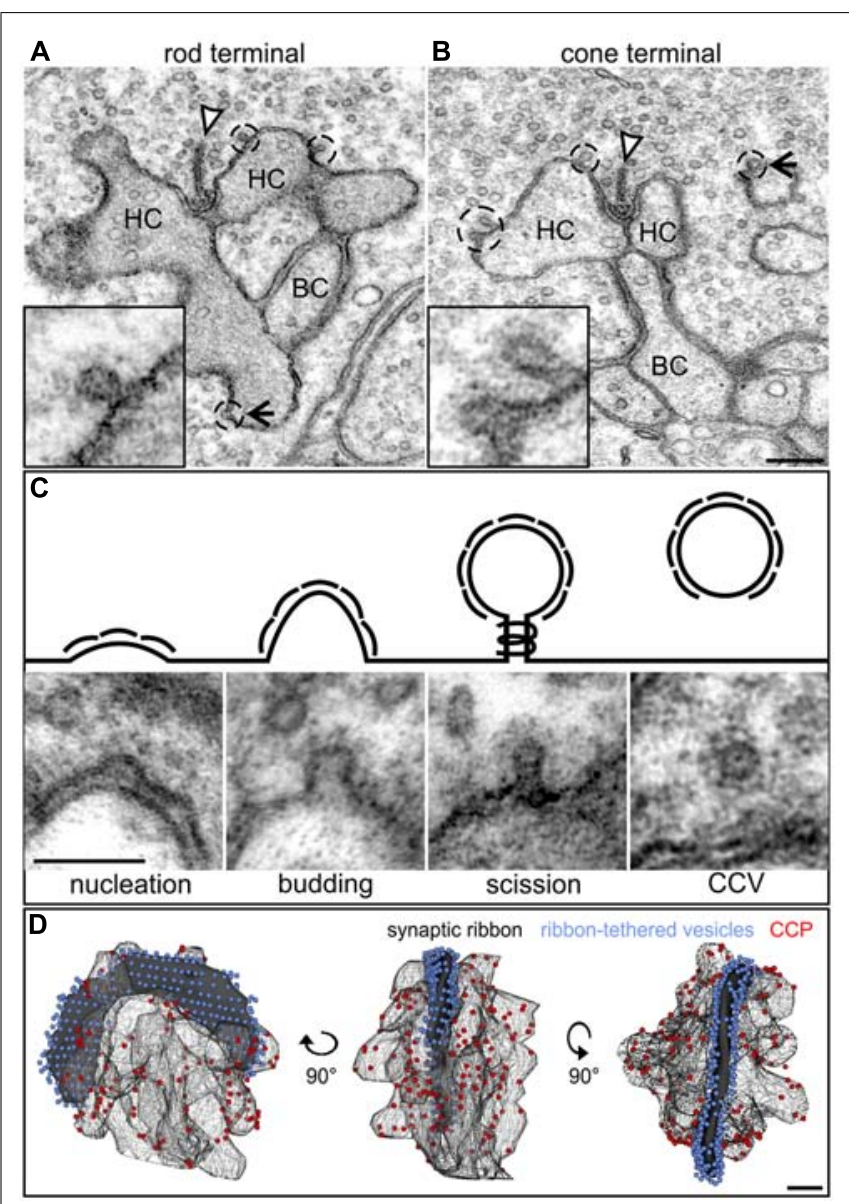

FIGURE 3 | Ultrastructural analysis of Clathrin-mediated endocytosis in photoreceptor ribbon synapses in a $\mathbf{3} \mathbf{h}$ light adapted retina. (A,B) Representative electron micrographs of a rod (A) and cone photoreceptor (B) terminal in the light adapted mouse retina. Clathrin-coated pits (CCP) budding off from the plasma membrane are demarcated with dashed lines and magnified in the insets. Arrowheads point to synaptic ribbons; arrows to CCP remote from synaptic ribbons. (C) Exemplary electron micrographs of Clathrin-coated structures from the different stages of Clathrin-mediated endocytosis (schematized above) in rod photoreceptor terminals. (D) 3D-reconstruction of a light adapted rod photoreceptor terminal viewed from different angles. The horseshoe-shaped synaptic ribbon (black) bends around the postsynaptic invaginations of bipolar and horizontal cell processes. Ribbon-tethered vesicles are shown in blue, CCP are shown in red. HC, horizontal cell; BC, bipolar cell. Scale bar in $\mathbf{B}$ (for $\mathbf{A}, \mathbf{B}$ ): $200 \mathrm{~nm}$, in C: $100 \mathrm{~nm}$, in D: $200 \mathrm{~nm}$.

to distinguish between rod and cone photoreceptor terminals, we additionally performed the stainings on Rac3-EGFP retinae which, express eGFP in the cone photoreceptor terminals (Figure 4D). Endophilin1, and most interestingly also Synaptojanin1 (Figures 4C,D) which had been virtually undetectable in the OPL in the light adapted retinae (Figures 2D,E) redistributed in the photoreceptor synaptic terminals similar to Dynamin3 after prolonged dark exposure. For Amphiphysin1 scattered immunofluorescent puncta could be detected, whereas Clathrin LC, AP180, and hsc70 were never observed to aggregate after prolonged dark periods (data not shown). The quantification of Dynamin3, Endophilin1, and Synaptojanin1 puncta in stretches of $90 \mu \mathrm{m}$ OPL after the different durations of dark exposure is shown in Figure 4B. After 1 and 15 min of dark adaptation, immunofluorescent puncta for Dynamin3, Endophilin1, and Synaptojanin 1 were rarely found, while the number of puncta markedly increased for all three proteins after 3 and $15 \mathrm{~h}$ of dark adaptation (Figure 4B). After $3 \mathrm{~h}$ of dark adaptation, almost all rod photoreceptor terminals contained one or two Dynamin3, Endophilin1, and Synaptojanin1 immunofluorescent puncta (Figure 4C). In the cone photoreceptor terminals, a punctate staining of the three endocytotic proteins was more frequent after $15 \mathrm{~h}$ of dark adaptation, with a higher number of puncta per terminal than in rod photoreceptor terminals (Figure $4 \mathbf{D}$ ). $3 \mathrm{D}$ reconstructions confirmed the localization of immunofluorescent puncta within the cone photoreceptor terminals (Figure 4D).

\section{PUNCTATE COLOCALIZATION OF DYNAMIN3, ENDOPHILIN1, AND SYNAPTOJANIN1 IN PHOTORECEPTOR SYNAPTIC TERMINALS AFTER PROLONGED DARK EXPOSURE}

Dynamin3, Endophilin1, and Synaptojanin1 displayed a punctate staining in the photoreceptor synaptic terminals after prolonged dark exposure (Figure 4). To test for colocalization of the three endocytotic proteins, we performed double labeling experiments for Dynamin3/Endophilin1, and Dynamin3/Synaptojanin1 (Figures 5A,B). Colocalization of Dynamin3 with Endophilin1 and Synaptojanin1, respectively, was apparent, although not complete, since single Dynamin3-, Endophilin1-, and Synaptojanin1positive puncta were detected as well (Figures 5A,B; arrows). The percentage of Dynamin3 puncta that colocalized with Endophilin1 and Synaptojanin 1 puncta increased with increasing periods of dark exposure (Figure 5C). At $15 \mathrm{~h}$ dark adaptation there was almost complete colocalization of the three endocytotic proteins (Figure 5C).

\section{ULTRASTRUCTURAL CHARACTERIZATION OF AN ENDOCYTOTIC VESICLE CLUSTER IN DARK ADAPTED PHOTORECEPTOR TERMINALS}

To analyze the presynaptic localization of the punctate immunoreactivity for Dynamin3, Endophilin1, and Synaptojanin1, we first triple labeled cryostat sections of $3 \mathrm{~h}$ dark adapted BL/6 retinae for Calbindin (green; labels horizontal cells and their invaginations into photoreceptor terminals), Dynamin3 (red), and Piccolino, a ribbon-specific Piccolo variant labeling the synaptic ribbon (blue; Regus-Leidig etal., 2013; Figure 6A). The merge of the three stainings shows that Dynamin3 puncta localize in the periactive zone close to the horseshoe shaped synaptic ribbons as well as the tips of horizontal cell processes (Figure 6A; arrows). To characterize this putative endocytotic hot spot at the ultrastructural level, we next performed pre-embedding immunoelectron microscopy on $3 \mathrm{~h}$ dark adapted BL/6 retinae with antibodies against Dynamin3, Endophilin1, Synaptojanin1 (Figures 6B-E), and Clathrin (data not shown). We also tried post-embedding immunoelectron microscopy, which unfortunately did not satisfactorily work in our hands. In rod photoreceptor terminals, immunoreactivity for Dynamin3, Endophilin1, Synaptojanin1 concentrated in the vicinity of the postsynaptic invaginations and the synaptic ribbon (Figures 6B-E; dashed circles). As expected, Clathrin LC immunoreactivity was diffusely distributed 

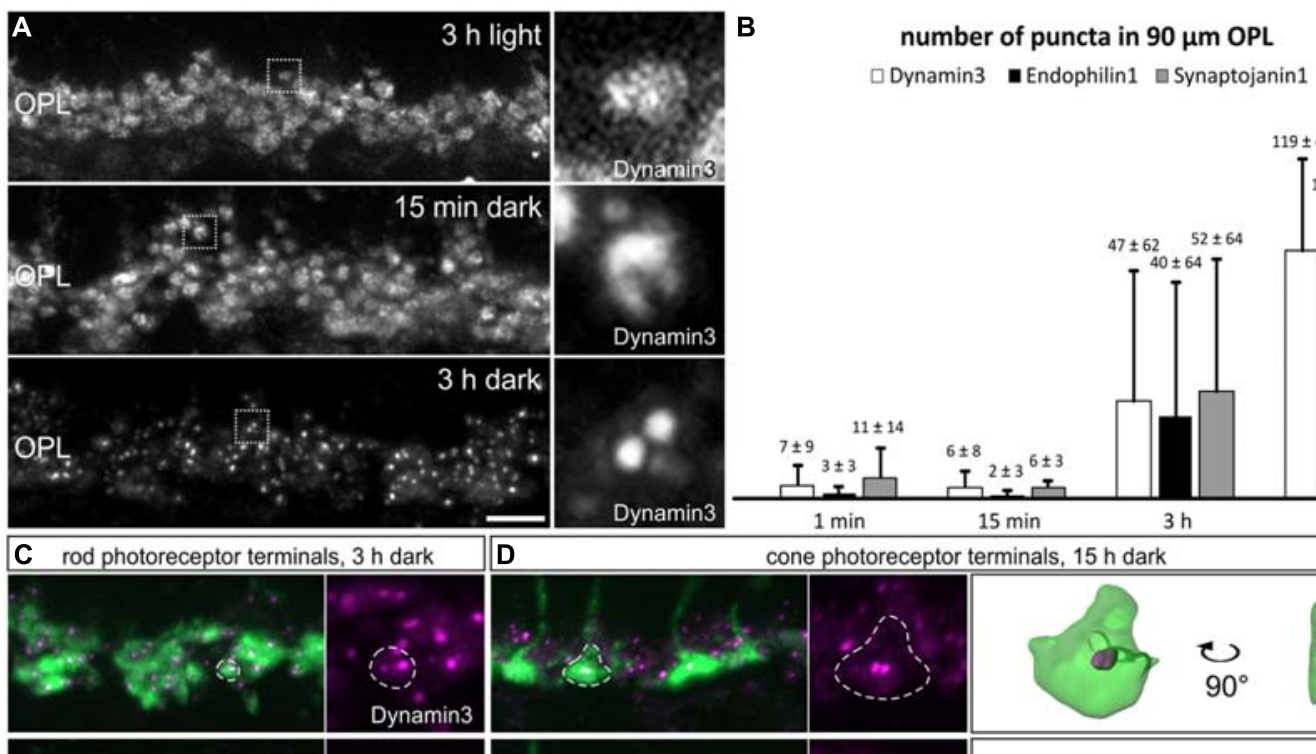

cone photoreceptor terminals, $15 \mathrm{~h}$ dark
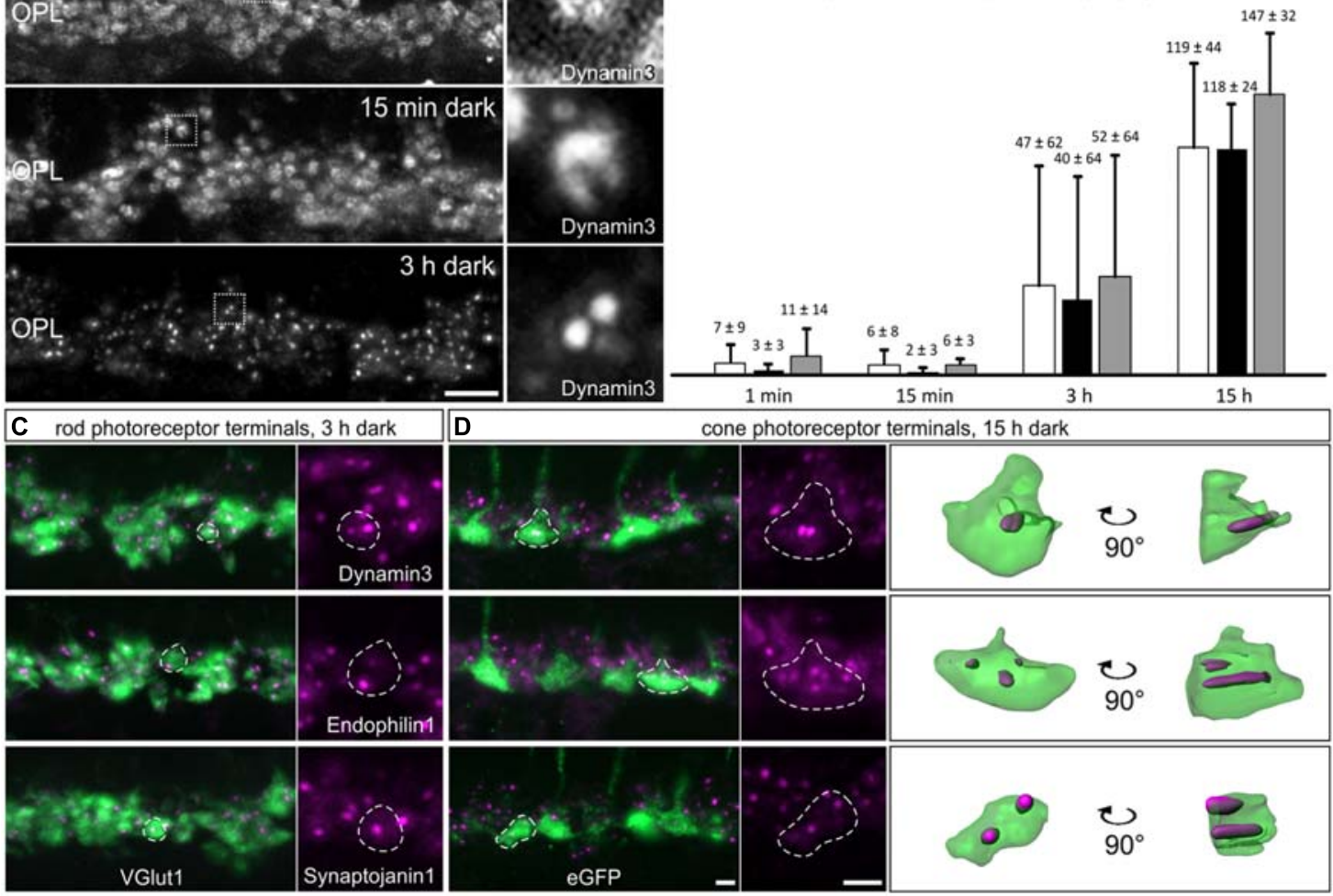

FIGURE 4 | Redistribution of endocytotic proteins in $1 \mathrm{~min}, 15 \mathrm{~min}, 3 \mathrm{~h}$, and $15 \mathrm{~h}$ dark adapted photoreceptor terminals. (A) Outer plexiform layer (OPL) of $\mathrm{C} 57 \mathrm{BL} / 6 \mathrm{JRj}$ retinae after different periods of dark adaptation labeled with an antibody against Dynamin3. Single synaptic terminals are shown in high magnification. (B) Quantification of immunofluorescent Dynamin3-, Endophilin1-, and Synaptojanin1 puncta in the OPL after different durations of dark adaptation. (C) Images of rod photoreceptor terminals of C57BL/6JRj retina after $3 \mathrm{~h}$ of dark adaptation stained with antibodies against VGluT1 (green) and Dynamin3, Endophilin1, or Synaptojanin1 (magenta). (D) Images of cone photoreceptor terminals of Rac3-EGFP retina after $15 \mathrm{~h}$ of dark adaptation stained with antibodies against GFP (green) and Dynamin3, Endophilin1, or Synaptojanin1 (magenta). Single synaptic terminals are delineated with dashed lines. Values in $\mathbf{B}$ are means \pm SD $(n=4$ retinae from 4 mice, 3 images per retina). Scale bar in $\mathbf{A}: 10 \mu \mathrm{m}$, in $\mathbf{D}$ (for $\mathbf{C}, \mathbf{D}): 2 \mu \mathrm{m}$. throughout the photoreceptor synaptic terminals, confirming the light microscopical absence of Clathrin LC in these endocytotic hot spots (data not shown). Sometimes, presumptive vesicles were visible in the labeled region (Figure $6 \mathrm{C}$ ), but most of the times the intense staining obscured any underlying structures. Therefore, we analyzed dark adapted BL/6 retinae with conventional electron microscopy for the presence of conspicuous vesicle clusters in the vicinity of the synaptic ribbon (Figure 7).

While we could not detect any obvious ultrastructural changes in rod and cone photoreceptor terminals comparing light and shortly dark exposed retinae ( 1 and $15 \mathrm{~min}$ ), we observed striking accumulations of electron-dense vesicles (dashed circles) in the vicinity of synaptic ribbons (arrowheads) and the postsynaptic elements after $3 \mathrm{~h}$, and $15 \mathrm{~h}$ (data not shown) of dark exposure in rod photoreceptor terminals (Figure 7A). These electron-dense vesicles could also be observed in cone terminals, albeit less frequently. Occasionally, these vesicle clusters were seen to emanate from the plasma membrane at the rod postsynaptic invaginations suggesting an endocytotic origin (Figure 7B; arrows). The closely connected vesicles in the cluster (Figure 7B; inset) were smaller than synaptic vesicles (mean diameter of electron-dense vesicles: $28.8 \pm 5.2 \mathrm{~nm}, \mathrm{SD}, n=1100$; mean diameter of synaptic vesicles: $37.3 \pm 3.8 \mathrm{~nm}, \mathrm{SD}, n=1100)$. 3D reconstructions of prolonged dark adapted rod photoreceptor terminals revealed the great extent of the clusters of electron-dense endocytotic vesicles (CEV; green; Figure $7 \mathrm{C}$ ) and confirmed their localization at the periactive zone and their connection to the plasma membrane. The vesicle numbers in 3D reconstructed CEV from seven photoreceptor terminals after $3 \mathrm{~h}$ of dark adaptation ranged from 291 to 1371 vesicles (mean: $695 \pm 381, \mathrm{SD}$ ). CEV were also present in photoreceptor terminals of dark adapted Balb/c mice and Dark Agouti rats (data not shown), excluding a strain or species specific phenomenon. Of note, we never observed CEV in ON bipolar cell terminals of light or dark adapted retinae, corroborating the finding that Dynamin3 did not redistribute in the ON bipolar cell terminals following light or dark adaptation.

Finally, the number of CCP determined from single ultrathin sections (Figure 7C; red spheres) increased with increasing periods of dark exposure, reaching highest numbers in the $15 \mathrm{~h}$ dark adapted state: light: $8.4 \pm 2.8 ; 1$ min dark: $7.7 \pm 2.7 ; 15$ min dark: $13.2 \pm 2.9 ; 3$ h dark: $13.9 \pm 4.1 ; 15$ h dark: $15.0 \pm 3.9 ; \operatorname{SD}(n=50$ 

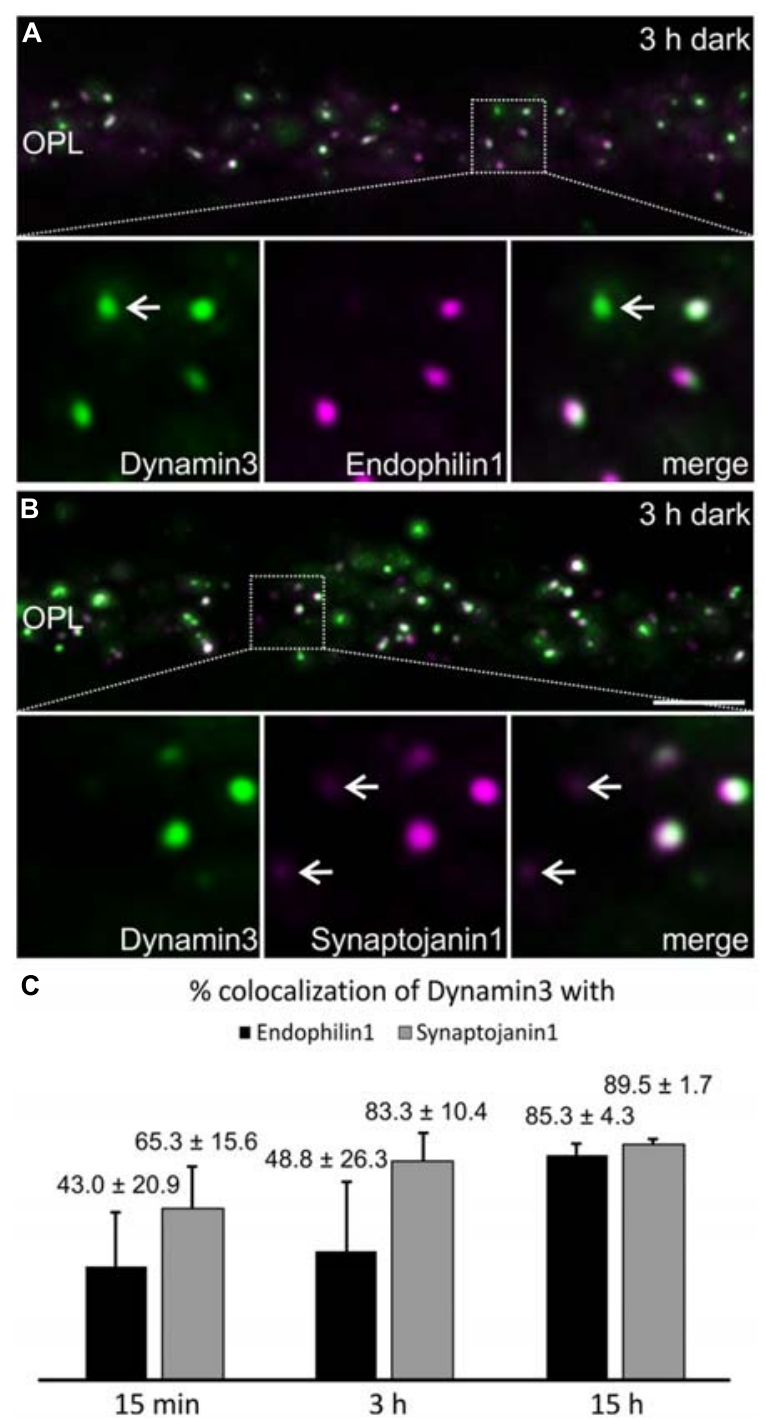

FIGURE 5 | Colocalization of Dynamin3 with Endophilin1 and Synaptojanin 1 in 15 min, 3 h, and 15 h dark adapted OPL. (A,B) Outer plexiform layer (OPL) of C57BL/6JRj retinae after $3 \mathrm{~h}$ of dark adaptation labeled with antibodies against Dynamin3 (green) and Endophilin1 (A) or Synaptojanin1 (B; magenta). Arrows in the high power views point to individual, non-colocalizing puncta. (C) Quantification of the colocalization of Dynamin3 puncta with Endophilin1 and Synaptojanin1 in the OPL after $15 \mathrm{~min}, 3 \mathrm{~h}$, and $15 \mathrm{~h}$ of dark adaptation. Values are means $\pm \operatorname{SD}(n=3$ retinae from 3 mice, 3 images per retina). Scale bar in $\mathbf{B}$ (for $\mathbf{A}, \mathbf{B}$ ): $5 \mu \mathrm{m}$.

terminals per retina; three retinae from three animals per adaptation state). It is important to note that, comparable to the light adapted state (Figure 3), also in the prolonged dark adapted retinae, CCP were not enriched around the ribbon synaptic site but evenly distributed along the plasma membrane of the postsynaptic complex (Figure 7C).

\section{CEV FORMATION IN PHOTORECEPTOR TERMINALS IN THE ABSENCE OF FUNCTIONAL BASSOON}

Clusters of electron-dense endocytotic vesicles were sometimes seen in close association with vesicles at the synaptic ribbon

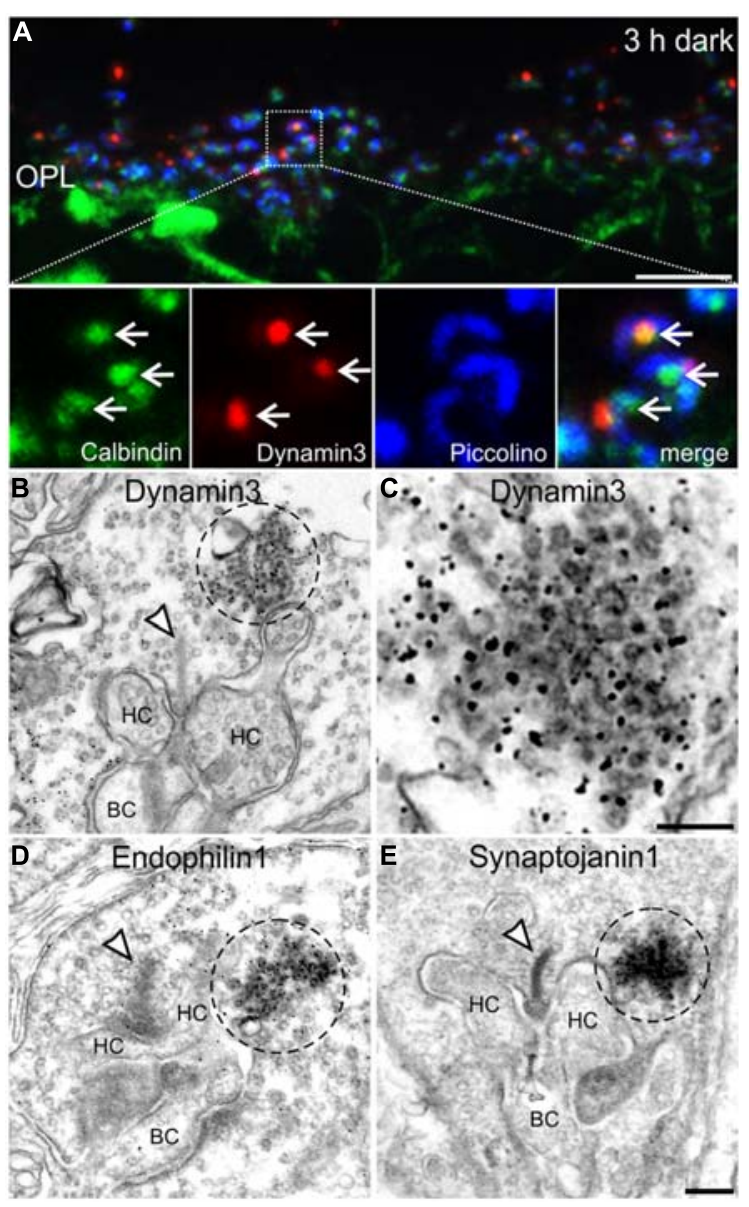

FIGURE 6 | Presynaptic localization of Dynamin3, Endophilin1, and Synaptojanin $\mathbf{1}$ in $\mathbf{3} \mathbf{~ h}$ dark adapted rod photoreceptor terminals. (A) Outer plexiform layer (OPL) of C57BL/6JRj retina after $3 \mathrm{~h}$ of dark adaptation labeled with antibodies against Calbindin (green), Dynamin3 (red), and Piccolino (blue). Arrows point to adjacent Calbindin- and Dynamin3-puncta. (B-E) Pre-embedding immunoelectron micrographs of $3 \mathrm{~h}$ dark adapted rod photoreceptor terminals stained with antibodies against Dynamin3 (B,C), Endophilin1 (D), and Synaptojanin1 (E). Immunoreactivity is encircled with dashed lines; arrowheads point to synaptic ribbons. HC, horizontal cell; BC, bipolar cell. Scale bar in A: $5 \mu \mathrm{m}$, in $\mathbf{D}$ (for $\mathbf{B}, \mathbf{D}, \mathbf{E}): 200 \mathrm{~nm}$, in $\mathbf{C}: 100 \mathrm{~nm}$.

(Figure 7D; arrowhead), hinting at a contribution of the synaptic ribbon to vesicle retrieval as postulated previously (Schmitz, 2009; Schwarz et al., 2011; Wahl et al., 2013). To analyze the importance of the synaptic ribbon for the formation of CEV, we made use of the Bassoon mutant mouse (Bsn $\Delta \mathrm{Ex} 4 / 5)$, which possesses detached, free floating photoreceptor synaptic ribbons due to a missing interaction of the anchor molecule Bassoon with RIBEYE (Dick et al., 2003; tom Dieck et al., 2005; Regus-Leidig et al., 2010b). Despite the absence of active zone anchored ribbons, punctate hotspots of Dynamin3 immunoreactivity were present in the photoreceptor terminals of the Bassoon mutant mouse after prolonged dark exposure (Figure 8A). Electron microscopy confirms the presence of CEV and, most importantly, the clear spatial separation of CEV and free ribbons the Bassoon mutant photoreceptor terminals (Figures 8B,C). 


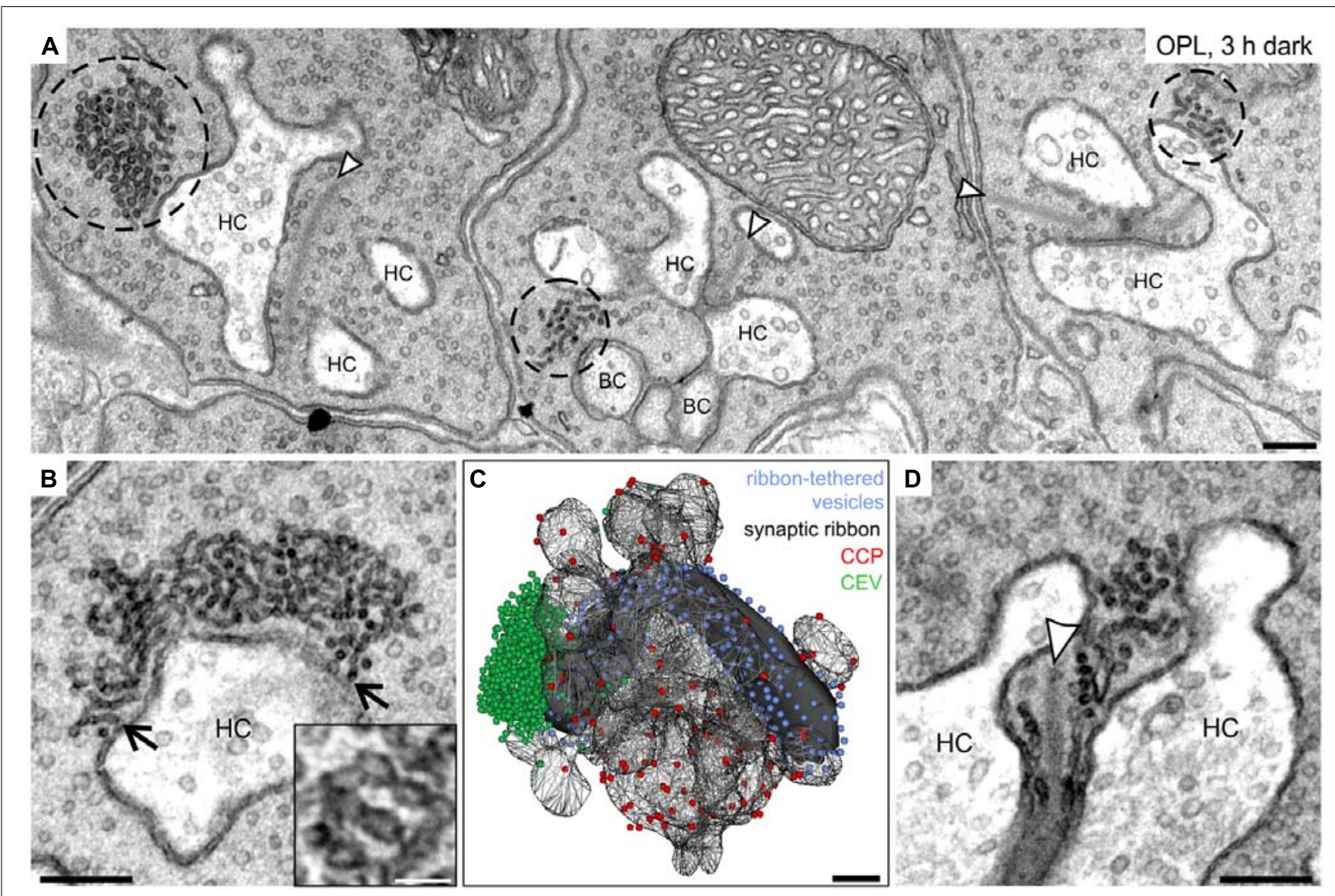

FIGURE 7 | Ultrastructural analysis of clusters of electron-dense endocytotic vesicles in $\mathbf{3} \mathbf{~ h}$ dark adapted rod photoreceptor terminals. (A) Electron micrograph of outer plexiform layer (OPL), showing clusters of electron dense endocytotic vesicles (CEV; encircled with dashed lines) in three neighboring rod photoreceptor terminals of a $3 \mathrm{~h}$ dark adapted C57BL/6JRj retina. (B) Representative electron micrograph of a rod photoreceptor terminal in the $3 \mathrm{~h}$ dark adapted retina. Arrows point to single vesicles of the CEV emanating from the plasma membrane at the postsynaptic invaginations. The high magnification inset shows the membranous connection between individual vesicles of a CEV.

(C) 3D-reconstruction of a $3 \mathrm{~h}$ dark adapted rod photoreceptor terminal. The horseshoe-shaped synaptic ribbon (black) bends around the postsynaptic invaginations of bipolar and horizontal cells. Ribbon-tethered vesicles are shown in blue, Clathrin-coated pits (CCP) in red, and CEV in green.

(D) Electron micrograph of a $3 \mathrm{~h}$ dark adapted rod photoreceptor terminal showing CEV in close association with vesicles tethered to the synaptic ribbon. Arrowheads point to synaptic ribbons. HC, horizontal cell; $\mathrm{BC}$, bipolar cell. Scale bar in A-D: $200 \mathrm{~nm}$, inset in B: $50 \mathrm{~nm}$.

\section{DISCUSSION}

In the present study, we analyzed the presynaptic localization of the endocytotic proteins Clathrin LC, AP180, Endophilin1, Amphiphysin1, Synaptojanin1, Dynamin3, and hsc70 at retinal ribbon synapses as a function of synaptic activity (light/dark). We found an adaptation- and thus activity-dependent redistribution of Dynamin3, Endophilin1, and Synaptojanin1 in rod and cone photoreceptor ribbon synapses. Figure 9 summarizes the main results of our study:

\section{LIGHT/LOW PHOTORECEPTOR SYNAPTIC ACTIVITY}

In light adapted photoreceptor synapses, CME is the predominant mode of endocytosis. Due to low rates of endocytosis in the periactive zone, the majority of Clathrin LC, Dynamin3, Endophilin1, and Synaptojanin1 was diffusely distributed in the cytoplasm (Figure 9A). In line with minimal synaptic activity, we found the lowest number of CCP in photoreceptor synaptic terminals of light adapted retinae.

\section{SHORT DARKNESS/HIGH PHOTORECEPTOR SYNAPTIC ACTIVITY (1 TO $15 \mathrm{~min}$ )}

In shortly dark exposed photoreceptor ribbon synapses, Clathrin LC, Dynamin3, Endophilin1, and Synaptojanin1 concentrated in the periactive zone surrounding the photoreceptor synaptic ribbon complex. This most likely reflects increased membrane turnover rates in conjunction with increased synaptic activity, a finding which is in agreement with the concept of periactive zone endocytosis in photoreceptor ribbon synapses (Wahl et al., 2013). In line with the assumption of high sustained photoreceptor synaptic activity in darkness, the number of CCP increased in the photoreceptor terminals following dark exposure.

\section{PROLONGED DARKNESS/HIGH PHOTORECEPTOR SYNAPTIC ACTIVITY (3 TO 15 h)}

After prolonged dark exposure, the number of CCP in the photoreceptor terminals was highest, and an additional mode of endocytosis involving Dynamin3, Endophilin1, and 


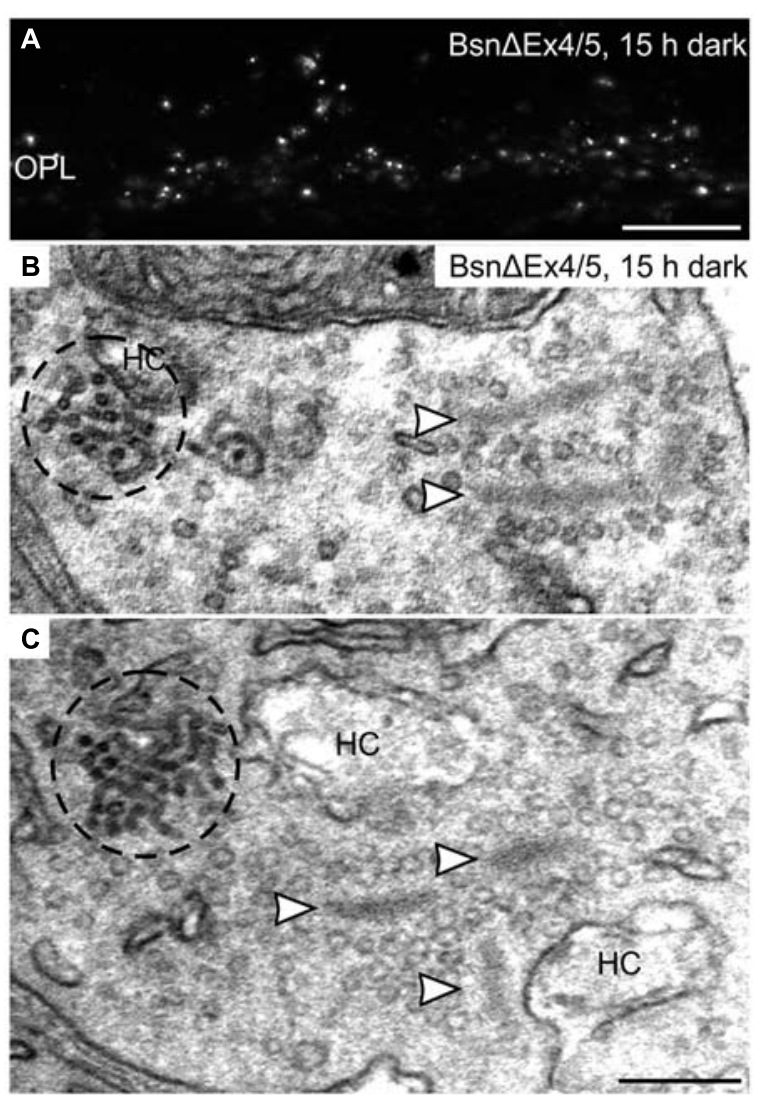

FIGURE 8 | Clusters of electron-dense endocytotic vesicles in $\mathbf{1 5}$ h dark adapted Bsn $\Delta$ Ex $4 / 5$ photoreceptor terminals. (A) Outer plexiform layer $(\mathrm{OPL})$ of a $15 \mathrm{~h}$ dark adapted Bsn $\Delta \mathrm{Ex} 4 / 5$ retina labeled with an antibody against Dynamin3. (B,C) Electron micrographs of rod photoreceptor terminals in a $15 \mathrm{~h}$ dark adapted Bsn $\Delta \mathrm{Ex} 4 / 5$ retina. Clusters of electron dense endocytotic vesicles (CEV) are encircled with dashed lines; arrowheads point to synaptic ribbons. HC, horizontal cell. Scale bar in $\mathbf{A}$ $10 \mu \mathrm{m}$, in $\mathbf{C}$ (for $\mathbf{B}, \mathbf{C})$ : $200 \mathrm{~nm}$.

Synaptojanin 1 occurred. The latter resulted in the uptake of membranously connected, electron-dense endocytotic vesicles (CEV; Figure 9C).

Both types of photoreceptors showed similar activitydependent protein redistribution and ultrastructure of CEV. Differences in temporal appearance (CEV in rod photoreceptor terminals occured earlier than in cone photoreceptor terminals) and number of CEV per terminal (one or two CEV in rod photoreceptor terminals, more than two CEV in cone photoreceptor terminals) possibly reflect the different functional demands on rod and cone photoreceptors.

Clathrin LC, AP180, and hsc70 are involved in the formation and degradation of the Clathrin coat (for review, see Edeling etal., 2006; McMahon and Boucrot, 2011). The absence of these proteins in the $\mathrm{CEV}$, together with the ultrastructural lack of Clathrin coats associated with the CEV, corroborate a Clathrin-independent formation of these endocytotic vesicles, and suggest that photoreceptor ribbon synapses possess, in addition to CME, a Clathrin-independent mode of vesicle retrieval initiated
$\Delta$ Clathrin LC $\Delta$ Dynamin3 $\triangle$ Endophilin1 $\Delta$ Synaptojanin1
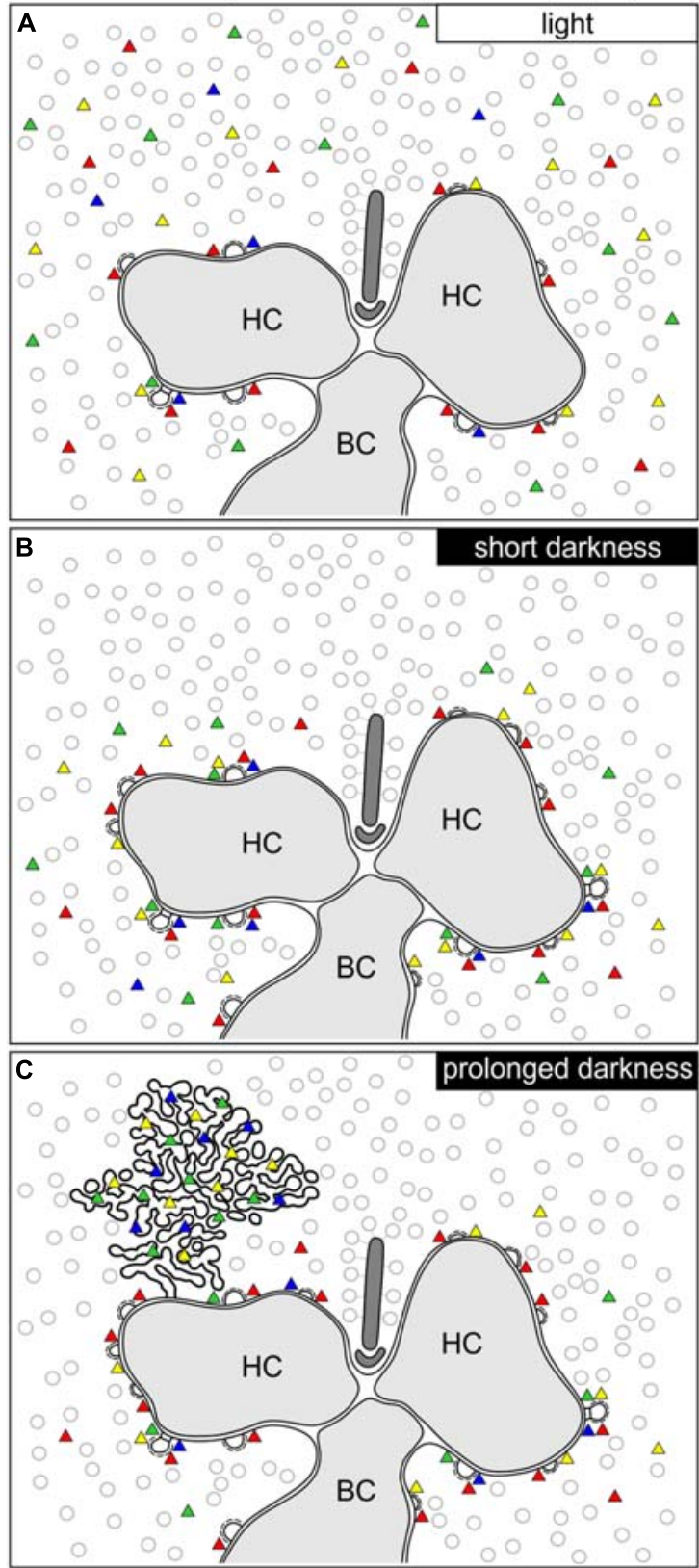

FIGURE 9 | Schematic summary of the hypothesized light/activity-dependent modes of endocytosis in photoreceptor terminals. (A-C) Photoreceptor ribbon synaptic site showing the postulated modes of endocytosis and the distribution of Dynamin3, Endophilin1, Synaptojanin1, and Clathrin LC (represented as colored triangles) in the light adapted state (A), after short periods of darkness (1 to 15 min; B), and after prolonged periods of darkness ( 3 to $15 \mathrm{~h} ; \mathbf{C}$ ). HC, horizontal cell; BC, bipolar cell.

during prolonged high synaptic activity. We could also show that the formation of $\mathrm{CEV}$ is not dependent on the presence of anchored synaptic ribbons, as CEV also formed in the Bsn $\triangle \mathrm{Ex} 4 / 5$ mouse retina, in which photoreceptor synaptic ribbons are not membrane-anchored but free floating in the synaptic terminals. 
Wahl etal. (2013) reported punctate staining in rod photoreceptor terminals with an antibody against Clathrin heavy chain (HC), resembling the punctate staining we observed for Dynamin3, Endophilin1, and Synaptojanin1 after prolonged dark exposure. However, these puncta do not correspond to the CEV shown in our study. First, the Clathrin HC puncta were found in light adapted photoreceptors, and second, they were located a large distance $(\sim 580 \mathrm{~nm})$ away from the synaptic ribbon. Furthermore, the authors interpret the Clathrin HC puncta to represent an endosomal compartment in the proximal region of the photoreceptor terminal (Wahl et al., 2013).

Organelles resembling the CEV have been described before in photoreceptors from various species as "tubular complexes/profiles/networks," "branched tubular structures," "accumulations of vesicle-like particles," "tubulovesicular structures," or "endoplasmatic reticulum (ER)-like structures” (Yamada, 1965; Samorajski, 1966; Lovas, 1971; Babai et al., 2010; Koch et al., 2011; López-del Hoyo et al., 2012). López-del Hoyo et al. (2012) hypothesized that these aggregates might be generated as "by-products in the bulk endocytosis," but no functional correlation with the level of synaptic activity was made. We are confident that the vesicle clusters we describe here are of endocytotic origin and do not represent ER for three reasons: (1) The clusters appear only after prolonged dark exposure, (2) they are connected to the presynaptic plasma membrane, and (3) they were never seen to be connected to other intracellular membrane structures.

The temporal appearance of CEV in dark adapted mouse photoreceptor terminals did not follow any general rule and was not directly correlated with the duration of dark exposure (see high standard deviation in Figure 4B). So far, we can therefore only speculate about the trigger and functional significance of CEV formation. At central nerve terminals, activity-dependent bulk endocytosis as a second vesicle retrieval mode has been reported in reaction to increased stimulation (Clayton et al., 2008; Dittman and Ryan, 2009), and the phosphorylation of Dynamin was shown to act as a switch from CME to additional bulk endocytosis (Clayton et al., 2009). The presence of CEV in photoreceptor terminals might therefore represent bulk endocytosis after prolonged high synaptic activity. However, bulk endocytosis, as also reported for bipolar cell ribbon synapses of the goldfish retina, involves the uptake of large bites of membrane with subsequent generation of synaptic vesicles (LoGiudice and Matthews, 2007). To our knowledge, bulk endocytosis consisting of individual, membranously connected vesicles has never been reported so far. CEV might represent a form of bulk vesicle retrieval without detour via endosomal intermediates for the rapid formation of releasable vesicles in continuously active photoreceptors, especially during sustained maximal activity (Rea et al., 2004). Although we sometimes found vesicles and/or tubules of CEV in close vicinity to synaptic ribbons, we doubt that $\mathrm{CEV}$ provide a direct reloading of the synaptic ribbon with new synaptic vesicles. In the majority of ultrathin sections as well as in all 3D reconstructions from rod terminals, $\mathrm{CEV}$ were localized at the presynaptic plasma membrane some distance away from the synaptic ribbon.

Curiously, although Dynamin as the required mechanoenzyme for the final fission step in vesicle formation is present on $\mathrm{CEV}, \mathrm{CEV}$ are not accumulations of free vesicles but aggregates of membranously connected vesicles. In the absence of functional Dynamin 1 (and 3) similar branched tubular networks, albeit with Clathrin-coats, were detected at mouse conventional chemical synapses (Ferguson et al., 2007; Raimondi et al., 2011). Therefore, instead of being a high-throughput endocytotic hotspot during sustained maximal activity, CEV might arise as an artifact during impaired vesicle retrieval, e.g., due to a limited availability of essential proteins or other factors during excessive synaptic activity. Since the GTPase Dynamin requires energy for its fission reaction (Ferguson and de Camilli, 2012), it is conceivable that accumulations of connected endocytotic vesicles might result during periods of energy shortage in the photoreceptor synaptic terminal. Since many steps in the presynapse are energyconsuming, e.g., priming of synaptic vesicles, the regulation of the $\left[\mathrm{Ca}^{2+}\right]_{i}$ by the plasma membrane Calcium-ATPase, and acidification of synaptic vesicles by the vacuolar $\mathrm{H}^{+}$-ATPase (reviewed in Südhof and Rizo, 2011; Harris et al., 2012), prolonged high synaptic activity may lead to metabolic stress in this cellular compartment.

However, although the impairment of compensatory endocytosis in conventional chemical synapses in the absence of Dynamin strongly impacts the availability of synaptic vesicles for exocytosis (Ferguson et al., 2007; Raimondi et al., 2011), we doubt that $\mathrm{CEV}$ as a putative sign of arrest in vesicle recycling would have any perturbing effect on photoreceptor synaptic transmission. The percentage of "retained" vesicles in a CEV in relation to the number of free synaptic vesicles in the presynaptic cytoplasm is less than $10 \%$ (judged from the vesicle numbers counted in serial sections of three 3D reconstructed dark adapted rod photoreceptor terminals), and thus would hardly limit the availability of releasable vesicles.

As in the photoreceptor terminals, also in the ON bipolar cell terminals we detected all analyzed endocytotic proteins, which is in line with the study by LoGiudice et al. (2009), who reported a Clathrin-mediated pathway for the retrieval of synaptic vesicles in mouse bipolar cell terminals. In contrast to the changing labeling pattern in photoreceptor terminals, the endocytotic proteins showed a diffuse distribution in bipolar cell terminals at all evaluated conditions. Moreover, at the ultrastructural level we never found endocytotic structures other than CCP, indicating CME as a major mode of endocytosis in mouse ON bipolar cell synapses in various activity states. The absence of CEV as putative membrane retrieval mechanism under prolonged activity may reflect the lower membrane turnover in bipolar cell terminals compared to photoreceptors synapses.

In conclusion, from our analysis of retinae in different adaptational states, we propose that the occurrence of CEV in the photoreceptor terminal reflects a stage of high synaptic activity. In future studies, it remains to be determined if CEV represent a special adaptation to the photoreceptors' need for continuous vesicle supply during high activity or rather a pathological artifact as a result of excessive stress.

\section{AUTHOR CONTRIBUTIONS}

Hanna Regus-Leidig and Michaela Fuchs designed research, conducted research, and analyzed data. Hanna Regus-Leidig, Michaela Fuchs and Johann Helmut Brandstätter wrote the paper. 


\section{ACKNOWLEDGMENTS}

Grant sponsor: Deutsche Forschungsgemeinschaft; Grant numbers BR 1643/4-2 (to Johann Helmut Brandstätter and Hanna Regus-Leidig) and BR 1643/5-1 (to Johann Helmut Brandstätter). We acknowledge support by Deutsche Forschungsgemeinschaft and Friedrich-Alexander-Universität Erlangen-Nürnberg (FAU) within the funding programme Open Access Publishing.

We thank N. Schröder for excellent technical assistance and Synaptic Systems for kindly providing many of the antibodies used in this study.

\section{REFERENCES}

Altrock, W. D., tom Dieck, S., Sokolov, M., Meyer, A. C., Sigler, A., Brakebusch, C., et al. (2003). Functional inactivation of a fraction of excitatory synapses in mice deficient for the active zone protein bassoon. Neuron 37, 787-800. doi: 10.1016/S0896-6273(03)00088-6

Babai, N., Morgans, C. W., and Thoreson, W. B. (2010). Calcium-induced calcium release contributes to synaptic release from mouse rod photoreceptors. Neuroscience 165, 1447-1456. doi: 10.1016/j.neuroscience.2009.11.032

Bai, L., Spiwoks-Becker, I., and Leube, R. E. (2006). Transcriptome comparison of murine wild-type and synaptophysin-deficient retina reveals complete identity. Brain Res. 1081, 53-58. doi: 10.1016/j.brainres.2006.01.080

Brandstätter, J. H., Löhrke, S., Morgans, C. W., and Wässle, H. (1996). Distributions of two homologous synaptic vesicle proteins, synaptoporin and synaptophysin, in the mammalian retina. J. Comp. Neurol. 370, 1-10. doi: 10.1002/(SICI)10969861(19960617)370:1<1::AID-CNE1 >3.0.CO;2-7

Brodin, L., Löw, P., and Shupliakov, O. (2000). Sequential steps in clathrinmediated synaptic vesicle endocytosis. Curr. Opin. Neurobiol. 10, 312-320. doi: 10.1016/S0959-4388(00)00097-0

Clayton, E. L., Anggono, V., Smillie, K. J., Chau, N., Robinson, P. J., and Cousin, M. A. (2009). The phospho-dependent dynamin-syndapin interaction triggers activitydependent bulk endocytosis of synaptic vesicles. J. Neurosci. 29, 7706-7717. doi: 10.1523/JNEUROSCI.1976-09.2009

Clayton, E. L., Evans, G. J. O., and Cousin, M. A. (2008). Bulk synaptic vesicle endocytosis is rapidly triggered during strong stimulation. J. Neurosci. 28, 66276632. doi: 10.1523/JNEUROSCI.1445-08.2008

Dick, O., Hack, I., Altrock, W. D., Garner, C. C., Gundelfinger, E. D., and Brandstätter, J. H. (2001). Localization of the presynaptic cytomatrix protein Piccolo at ribbon and conventional synapses in the rat retina: comparison with Bassoon. J. Comp. Neurol. 439, 224-234. doi: 10.1002/cne.1344

Dick, O., tom Dieck, S., Altrock, W. D., Ammermüller, J., Weiler, R., Garner, C. C., et al. (2003). The presynaptic active zone protein bassoon is essential for photoreceptor ribbon synapse formation in the retina. Neuron 37, 775-786. doi: 10.1016/S0896-6273(03)00086-2

Dittman, J., and Ryan, T. A. (2009). Molecular circuitry of endocytosis at nerve terminals. Annu. Rev. Cell Dev. Biol. 25, 133-160. doi: 10.1146/annurev.cellbio.042308.113302

Edeling, M. A., Smith, C., and Owen, D. (2006). Life of a clathrin coat: insights from clathrin and AP structures. Nat. Rev. Mol. Cell Biol. 7, 32-44. doi: $10.1038 / \mathrm{nrm} 1786$

Ferguson, S. M., Brasnjo, G., Hayashi, M., Wölfel, M., Collesi, C., Giovedi, S., et al. (2007). A selective activity-dependent requirement for dynamin 1 in synaptic vesicle endocytosis. Science 316, 570-574. doi: 10.1126/science. 1140621

Ferguson, S. M., and de Camilli, P. (2012). Dynamin, a membrane-remodelling GTPase. Nat. Rev. Mol. Cell Biol. 13, 75-88. doi: 10.1038/nrm3266

Gray, E. G., and Pease, H. L. (1971). On understanding the organisation of the retinal receptor synapses. Brain Res. 35, 1-15. doi: 10.1016/0006-8993(71)90591-9

Grossman, G. H., Ebke, L. A., Beight, C. D., Jang, G.-F., Crabb, J. W., and Hagstrom, S. A. (2013). Protein partners of dynamin-1 in the retina. Vis. Neurosci. 30, 129-139. doi: 10.1017/S0952523813000138

Harris, J. J., Jolivet, R., and Attwell, D. (2012). Synaptic energy use and supply. Neuron 75, 762-777. doi: 10.1016/j.neuron.2012.08.019

Haucke, V., Neher, E., and Sigrist, S. J. (2011). Protein scaffolds in the coupling of synaptic exocytosis and endocytosis. Nat. Rev. Neurosci. 12, 127-138. doi: $10.1038 / \mathrm{nrn} 2948$
Heidelberger, R., Thoreson, W. B., and Witkovsky, P. (2005). Synaptic transmission at retinal ribbon synapses. Prog. Retin. Eye Res. 24, 682-720. doi: 10.1016/j.preteyeres.2005.04.002

Holzhausen, L. C., Lewis, A. A., Cheong, K. K., and Brockerhoff, S. E. (2009). Differential role for synaptojanin 1 in rod and cone photoreceptors. J. Comp. Neurol. 517, 633-644. doi: 10.1002/cne.22176

Jockusch, W. J., Praefcke, G. J., McMahon, H. T., and Lagnado, L. (2005). Clathrindependent and clathrin-independent retrieval of synaptic vesicles in retinal bipolar cells. Neuron 46, 869-878. doi: 10.1016/j.neuron.2005.05.004

Kachi, S., Yamazaki, A., and Usukura, J. (2001). Localization of caveolin-1 in photoreceptor synaptic ribbons. Invest. Ophthalmol. Vis. Sci. 42, 850-852.

Kim, H., Lee, T., Lee, J., Ahn, M., Moon, C., Wie, M. B., et al. (2006). Immunohistochemical study of caveolin-1 and -2 in the rat retina. J. Vet. Sci. 7, 101-104. doi: 10.4142/jvs.2006.7.2.101

Koch, D., Spiwoks-Becker, I., Sabanov, V., Sinning, A., Dugladze, T., Stellmacher, A., et al. (2011). Proper synaptic vesicle formation and neuronal network activity critically rely on syndapin I. EMBO J. 30, 4955-4969. doi: 10.1038/emboj.2011.339

Llobet, A., Gallop, J. L., Burden, J. J. E., Camdere, G., Chandra, P., Vallis, Y., et al. (2011). Endophilin drives the fast mode of vesicle retrieval in a ribbon synapse. J. Neurosci. 31, 8512-8519. doi: 10.1523/JNEUROSCI.6223-09.2011

LoGiudice, L., and Matthews, G. (2007). Endocytosis at ribbon synapses. Traffic 8, 1123-1128. doi: 10.1111/j.1600-0854.2007.00591.x

LoGiudice, L., Sterling, P., and Matthews, G. (2009). Vesicle recycling at ribbon synapses in the finely branched axon terminals of mouse retinal bipolar neurons. Neuroscience 164, 1546-1556. doi: 10.1016/j.neuroscience.2009.09.023

López-del Hoyo, N., Fazioli, L., López-Begines, S., Fernández-Sánchez, L., Cuenca, N., Llorens, J., et al. (2012). Overexpression of guanylate cyclase activating protein 2 in rod photoreceptors in vivo leads to morphological changes at the synaptic ribbon. PLoS ONE 7:e42994. doi: 10.1371/journal.pone. 0042994

Lovas, B. (1971). Tubular networks in the terminal endings of the visual receptor cells in the human, the monkey, the cat and the dog. Z. Zellforsch. 121, 341-357. doi: 10.1007/BF00337638

Matthews, G., and Fuchs, P. (2010). The diverse roles of ribbon synapses in sensory neurotransmission. Nat. Rev. Neurosci. 11, 812-822. doi: 10.1038/nrn2924

McMahon, H. T., and Boucrot, E. (2011). Molecular mechanism and physiological functions of clathrin-mediated endocytosis. Nat. Rev. Mol. Cell Biol. 12, 517-533. doi: 10.1038/nrm3151

Mercer, A. J., and Thoreson, W. B. (2011). The dynamic architecture of photoreceptor ribbon synapses: cytoskeletal, extracellular matrix, and intramembrane proteins. Vis. Neurosci. 28, 453-471. doi: 10.1017/S0952523811000356

Mühlhans, J., Brandstätter, J. H., and Gießl, A. (2011). The centrosomal protein pericentrin identified at the basal body complex of the connecting cilium in mouse photoreceptors. PLoS ONE 6:e26496. doi: 10.1371/journal.pone. 0026496

Nichols, B. J., and Lippincott-Schwartz, J. (2001). Endocytosis without clathrin coats. Trends Cell Biol. 11, 406-412. doi: 10.1016/S0962-8924(01)02107-9

Raimondi, A., Ferguson, S. M., Lou, X., Armbruster, M., Paradise, S., Giovedi, S., etal. (2011). Overlapping role of dynamin isoforms in synaptic vesicle endocytosis. Neuron 70, 1100-1114. doi: 10.1016/j.neuron.2011.04.031

Rao-Mirotznik, R., Harkins, A. B., Buchsbaum, G., and Sterling, P. (1995). Mammalian rod terminal: architecture of a binary synapse. Neuron 14, 561-569. doi: 10.1016/0896-6273(95)90312-7

Rea, R., Li, J., Dharia, A., Levitan, E. S., Sterling, P., and Kramer, R. H. (2004). Streamlined synaptic vesicle cycle in cone photoreceptor terminals. Neuron 41, 755-766. doi: 10.1016/S0896-6273(04)00088-1

Regus-Leidig, H., and Brandstätter, J. H. (2012). Structure and function of a complex sensory synapse. Acta Physiol. (Oxf.) 204, 479-486. doi: 10.1111/j.17481716.2011.02355.x

Regus-Leidig, H., Ott, C., Löhner, M., Atorf, J., Fuchs, M., Sedmak, T., et al. (2013). Identification and immunocytochemical characterization of piccolino, a novel piccolo splice variant selectively expressed at sensory ribbon synapses of the eye and ear. PLoS ONE 8:e70373. doi: 10.1371/journal.pone.0070373

Regus-Leidig, H., Specht, D., tom Dieck, S., and Brandstätter, J. H. (2010a). Stability of active zone components at the photoreceptor ribbon complex. Mol. Vis. 16, 2690-2700.

Regus-Leidig, H., tom Dieck, S., and Brandstätter, J. H. (2010b). Absence of functional active zone protein Bassoon affects assembly and transport of ribbon 
precursors during early steps of photoreceptor synaptogenesis. Eur. J. Cell Biol. 89, 468-475. doi: 10.1016/j.ejcb.2009.12.006

Regus-Leidig, H., tom Dieck, S., Specht, D., Meyer, L., and Brandstätter, J. H. (2009). Early steps in the assembly of photoreceptor ribbon synapses in the mouse retina: the involvement of precursor spheres. J. Comp. Neurol. 512, 814-824. doi: $10.1002 /$ cne. 21915

Royle, S. J., and Lagnado, L. (2010). Clathrin-mediated endocytosis at the synaptic terminal: bridging the gap between physiology and molecules. Traffic 11, 14891497. doi: 10.1111/j.1600-0854.2010.01104.x

Saheki, Y., and de Camilli, P. (2012). Synaptic vesicle endocytosis. Cold Spring Harb. Perspect. Biol. 4, a005645. doi: 10.1101/cshperspect.a005645

Samorajski, T. (1966). Structural organization of the retina in the tree shrew (Tupaia glis). J. Cell Biol. 28, 489-504. doi: 10.1083/jcb.28.3.489

Schmitz, F. (2009). The making of synaptic ribbons: how they are built and what they do. Neuroscientist 15, 611-624. doi: 10.1177/1073858409340253

Schneider, C. A., Rasband, W. S., and Eliceiri, K. W. (2012). NIH Image to ImageJ: 25 years of image analysis. Nat. Methods 9, 671-675. doi: 10.1038/nmeth.2089

Schwarz, K., Natarajan, S., Kassas, N., Vitale, N., and Schmitz, F. (2011). The synaptic ribbon is a site of phosphatidic acid generation in ribbon synapses. J. Neurosci. 31, 15996-16011. doi: 10.1523/JNEUROSCI.2965-11.2011

Sherry, D. M., and Heidelberger, R. (2005). Distribution of proteins associated with synaptic vesicle endocytosis in the mouse and goldfish retina. J. Comp. Neurol. 484, 440-457. doi: 10.1002/cne.20504

Spiwoks-Becker, I., Vollrath, L., Seeliger, M. W., Jaissle, G., Eshkind, L. G., and Leube, R. E. (2001). Synaptic vesicle alterations in rod photoreceptors of synaptophysindeficient mice. Neuroscience 107, 127-142. doi: 10.1016/S0306-4522(01)00345-1

Sterling, P., and Matthews, G. (2005). Structure and function of ribbon synapses. Trends Neurosci. 28, 20-29. doi: 10.1016/j.tins.2004.11.009

Südhof, T. C., and Rizo, J. (2011). Synaptic vesicle exocytosis. Cold Spring Harb. Perspect. Biol. 3, a005637. doi: 10.1101/cshperspect.a005637

tom Dieck, S., Altrock, W. D., Kessels, M. M., Qualmann, B., Regus, H., Brauner, D., et al. (2005). Molecular dissection of the photoreceptor ribbon synapse: physical interaction of Bassoon and RIBEYE is essential for the assembly of the ribbon complex. J. Cell Biol. 168, 825-836. doi: 10.1083/jcb.200408157

tom Dieck, S., and Brandstätter, J. H. (2006). Ribbon synapses of the retina. Cell Tissue Res. 326, 339-346. doi: 10.1007/s00441-006-0234-0
Van Hook, M. J., and Thoreson, W. B. (2012). Rapid synaptic vesicle endocytosis in cone photoreceptors of salamander retina. J. Neurosci. 32, 18112-18123. doi: 10.1523/JNEUROSCI.1764-12.2012

Wahl, S., Katiyar, R., and Schmitz, F. (2013). A local, periactive zone endocytic machinery at photoreceptor synapses in close vicinity to synaptic ribbons. J. Neurosci. 33, 10278-10300. doi: 10.1523/JNEUROSCI.504812.2013

Xi, Q., Pauer, G. J. T., Ball, S. L., Rayborn, M., Hollyfield, J. G., Peachey, N. S., et al. (2007). Interaction between the photoreceptor-specific tubby-like protein 1 and the neuronal-specific GTPase dynamin-1. Invest. Ophthalmol. Vis. Sci. 48, 2837-2844. doi: 10.1167/iovs.06-0059

Yamada, E. (1965). "Some observations on the membrane-limited structure within the retinal element," in Intracellular Membraneous Structure, eds S. Seno and E. V. Cowdry (Okayama: Japan Society Cell Biology), 49-63.

Zampighi, G. A., Schietroma, C., Zampighi, L. M., Woodruff, M., Wright, E. M., and Brecha, N. C. (2011). Conical tomography of a ribbon synapse: structural evidence for vesicle fusion. PLoS ONE 6:e16944. doi: 10.1371/journal.pone. 0016944

Conflict of Interest Statement: The authors declare that the research was conducted in the absence of any commercial or financial relationships that could be construed as a potential conflict of interest.

Received: 06 November 2013; accepted: 10 February 2014; published online: 25 February 2014.

Citation: Fuchs M, Brandstätter JH and Regus-Leidig H (2014) Evidence for a Clathrinindependent mode of endocytosis at a continuously active sensory synapse. Front. Cell. Neurosci. 8:60. doi: 10.3389/fncel.2014.00060

This article was submitted to the journal Frontiers in Cellular Neuroscience.

Copyright (c) 2014 Fuchs, Brandstätter and Regus-Leidig. This is an open-access article distributed under the terms of the Creative Commons Attribution License (CC BY). The use, distribution or reproduction in other forums is permitted, provided the original author(s) or licensor are credited and that the original publication in this journal is cited, in accordance with accepted academic practice. No use, distribution or reproduction is permitted which does not comply with these terms. 